\title{
Mastitis granulomatosa en centros de referencia en Medellín (Colombia), 2019. Estudio descriptivo
}

\author{
Granulomatous mastitis in reference centers in Medellin (Colombia), 2019. Descriptive \\ study
}

Óscar A. Bonilla-Sepulveda*

Servicio de Cirugía de Mama, Centro Oncológico de Antioquía, Medellín, Antioquía, Colombia

\begin{abstract}
Resumen
Objetivo: Describir los datos sociodemográficos, las manifestaciones clínicas, los estudios paraclínicos y el tratamiento en una serie de pacientes diagnosticadas de mastitis granulomatosa. Método: Estudio descriptivo, retrospectivo de una base de datos institucionales, entre el 1 de enero de 2016 y el 31 de diciembre de 2019. Se recopiló la información de registros médicos correspondiente a datos demográficos, clínicos y paraclínicos, y se analizaron utilizando estadística descriptiva. Resultados: La prevalencia en la muestra fue del $2.12 \%$, todos los casos fueron mujeres y de raza mestiza, la media de edad al momento del diagnóstico fue de 30.4 años y el motivo de consulta más frecuente fueron los cambios inflamatorios mamarios (12, 66.7\%), seguidos por una masa $(5,27.8 \%)$. La mayoría de las pacientes recibieron antibióticos como tratamiento inicial $(13,72.2 \%)$, terapia con esteroides $(8,44.4 \%)$ y metotrexato $(2,11.1 \%)$, mientras que 3 pacientes $(16.7 \%)$ se sometieron a cirugía. La resolución se dio en promedio en 7 meses, y 5 pacientes (27.8\%) tuvieron recurrencia. Conclusiones: La mastitis granulomatosa afecta a las mujeres en edad fértil, se presenta con cambios inflamatorios y dolor, tiende a presentar una evolución crónica con algunos episodios de recurrencia y hay resolución espontánea en casi todos los casos.
\end{abstract}

Palabras clave: Mastitis granulomatosa. Mama. Mastitis. Tratamiento.

\begin{abstract}
Objective: To describe the sociodemographic data, clinical manifestations, paraclinical and treatment, among the series of patients diagnosed with granulomatous mastitis. Method: A descriptive, retrospective study of an institutional database between January 1, 2016 to December 31, 2019. Data from medical records of demographic, clinical and paraclinical data were collected and analyzed using descriptive statistics. Results: The prevalence in the sample was $2.12 \%$, all cases were women, mixed race, the mean age at diagnosis was 30.4 years, the most frequent reason for consultation was inflammatory breast changes (12, 66.7\%), followed by mass (5, 27.8\%). Most of the patients received antibiotics as initial treatment $(13,72.2 \%)$, steroid therapy $(8,44.4 \%)$ and methotrexate $(2,11.1 \%)$, while 3 patients $(16.7 \%)$ underwent surgery. Resolution occurred on average in 7 months and 5 patients $(27.8 \%)$ had recurrence. Conclusions: Granulomatous mastitis affects women of childbearing age, presents with inflammatory changes and pain, tends to present a chronic evolution with some episodes of recurrence and spontaneous resolution in almost all cases.
\end{abstract}

Key words: Granulomatous mastitis. Breast. Mastitis. Treatment.

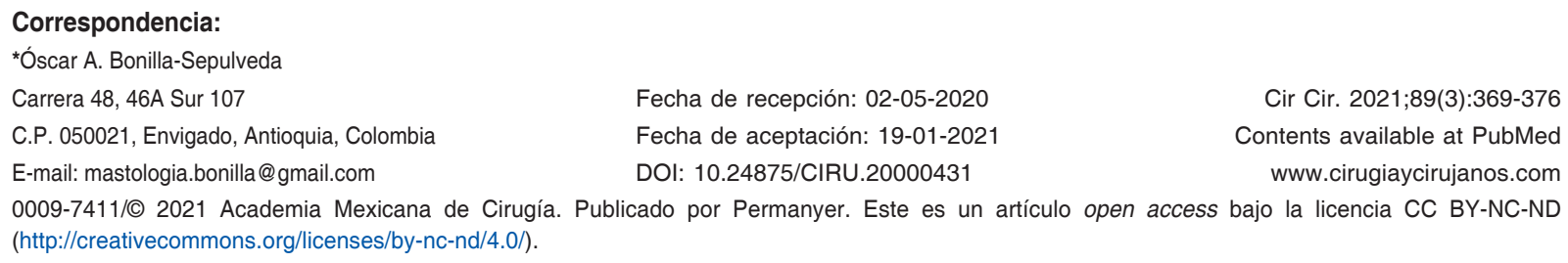




\section{Introducción}

La mastitis granulomatosa es una enfermedad inflamatoria de la mama, benigna y rara, descrita originalmente en 1972 por Kessler y Wolloch 1 . Su etiología no se conoce, pero la hipótesis más aceptada puede ser una reacción autoinmunitaria localizada en el seno $^{2-4}$.

Su verdadera prevalencia es desconocida. En un estudio de Baslaim, et al. ${ }^{5}$ con confirmación histopatológica se encontró en el 1.8\% de 1106 mujeres con enfermedad mamaria benigna, y en los Estados Unidos de Norteamérica se ha reportado una incidencia estimada de 2.4 por 100,000 mujeres, o del $0.37 \%$. Aunque hay reportes en todo el mundo, tiene una mayor predilección racial por las latinas y las mujeres asiáticas ${ }^{5}$.

Afecta principalmente a mujeres en edad fértil, aunque el rango de edad en la literatura es amplio (11-83 años) ${ }^{7-9}$. La mayoría de las pacientes tienen antecedentes recientes de embarazo y lactancia ${ }^{4,5,10}$.

La presentación clínica más común es una masa mamaria, de consistencia dura, con formación de abscesos, de predominio unilateral (la afectación bilateral es rara) $)^{11-13}$. Puede estar localizada en cualquier cuadrante, siendo más frecuente en la región subareolar, o puede presentarse de forma difusa. La paciente también puede presentar dolor, eritema, fistulización única o múltiple, hinchazón o linfadenopatía axilar ${ }^{14}$. La enfermedad puede ser agresiva localmente, con tasas de recurrencia reportadas del $16-50 \%{ }^{15-17}$.

El diagnóstico de mastitis granulomatosa solo puede confirmarse por histopatología. Las herramientas de diagnóstico incluyen la biopsia con aguja gruesa (BAG) con guía ecográfica ${ }^{3,18,19}$. Antes de 1980, la escisión quirúrgica de toda la lesión era más común ${ }^{4,19}$, pero en la actualidad se prefiere la $\mathrm{BAG}^{3,20}$.

La biopsia con aguja cortante, en su estudio histopatológico, se caracteriza por la presencia de granulomas no caseificantes de células gigantes multinucleados, limitados a lóbulos mamarios con microabscesos $^{21-23}$. La citología por aspiración con aguja fina a menudo revela abundancia de histiocitos epitelioides, en un fondo donde predominan los neutrófilos ${ }^{11,12}$.

El objetivo de este estudio fue hacer una revisión retrospectiva de los aspectos clínicos, el diagnóstico y el tratamiento de pacientes que asistieron a la consulta de mastología en tres clínicas de referencia en la ciudad de Medellín, con diagnóstico histopatológico de mastitis granulomatosa, entre el 1 de enero de 2016 y el 31 de diciembre de 2019.

\section{Método}

Estudio descriptivo retrospectivo de usuarias atendidas en la consulta de mastología de las clínicas Profamilia, Clínica Medellín y Clínica del Prado, en la ciudad de Medellín, entre el 1 de enero de 2016 y el 31 de diciembre de 2019. En total fueron atendidas 848 usuarias y se hizo una búsqueda de las historias clínicas con el código N61X Trastornos inflamatorios de la mama de la Clasificación Internacional de Enfermedades (CIE-10). Se identificaron 53 registros que correspondían con el diagnóstico de mastitis no puerperal, se creó una base de datos independiente para esta cohorte de pacientes y se realizó un análisis de subgrupo de los 18 casos con diagnóstico histopatológico de mastitis granulomatosa y con estudio microbiológico que descartara una infección bacteriana, tuberculosa o micótica. Se tomaron de la historia clínica los datos demográficos y las características clínicas y patológicas. Se incluyeron origen étnico, edad, historia de tabaquismo, fecha del último parto, características clínicas como motivo de consulta, hallazgos físicos, lado afectado, hallazgos en la ecografía y mamografía, tipos de tratamiento, como antibioticoterapia, esteroides o metotrexato, intervención quirúrgica, seguimiento y recurrencia.

Se establecieron tres criterios necesarios para el diagnóstico de mastitis granulomatosa:

- Cultivo negativo para microorganismos aerobios.

- Confirmación histopatológica, por BAG o tumorectomía, de granuloma no caseificante confinado a los lóbulos mamarios, no asociado a trauma ni posquirúrgico.

- Estudio de coloración de histoquímica negativo para Ziehl-Neelsen y plata-metenamina.

Se realizó un análisis descriptivo de las variables sociodemográficas y clínicas. Se calcularon frecuencias absolutas y porcentajes, promedio y desviación estándar, según la naturaleza y la distribución de las variables. Se validó la distribución normal usando la prueba de bondad de ajuste de Shapiro Wilk. Se realizó un análisis bivariado con la prueba ji al cuadrado para establecer la asociación entre resolución o recurrencia y modalidades de tratamiento. Para las pruebas de hipótesis se fijó un intervalo de confianza 


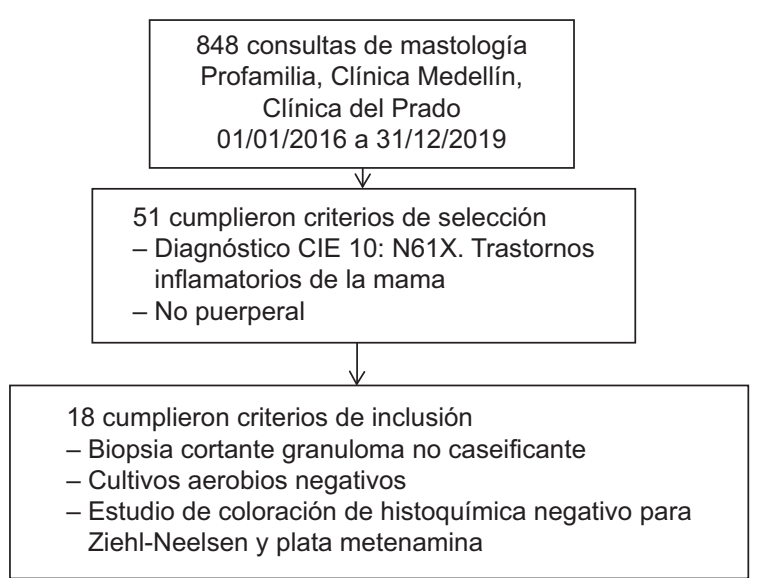

Figura 1. Flujograma de pacientes.

del $95 \%$ y un nivel de significación del 5\%. Se utilizó el software Epidat versión 3.1.

\section{Aspectos éticos}

Esta investigación fue sometida al comité de ética médica de la Clínica Profamilia, protocolo autorizado por dirección médica; investigación sin riesgo de acuerdo con la clasificación planteada en el Artículo 11 de la Resolución N. ${ }^{\circ} 008430$ de 1993 (expedida por el Ministerio de Salud de Colombia).

\section{Resultados}

Desde el 1 de enero de 2016 hasta el 31 de diciembre de 2019 fueron atendidas 848 pacientes con diagnóstico con el código N61X Trastornos inflamatorios de la mama de la $\mathrm{CIE}-10$. Se identificaron 53 con diagnóstico de mastitis no puerperal y 18 cumplieron los criterios de inclusión para mastitis granulomatosa; para esta cohorte, la prevalencia es del 2.12\% (Fig. 1).

\section{Características sociodemográficas y clínicas}

Las características sociodemográficas se muestran en la tabla 1. Todos los casos $(18,100 \%)$ fueron mujeres de raza mestiza, la mayoría atendidas en la Clínica Profamilia $(11,61.1 \%)$ y residentes en Medellín (10, 55.6\%). La edad media fue de 30.4 años (desviación estándar [DE]: 5.7; rango: 27-48). La mayoría de las pacientes $(17,94.4 \%)$ sin consumo de tabaco ni historia familiar de cáncer de mama (14, 77.8\%). La
Tabla 1. Características sociodemográficas

\begin{tabular}{|c|c|}
\hline Variable & $\begin{array}{c}\quad(n=18) \\
\text { Frecuencia (\%) }\end{array}$ \\
\hline \multicolumn{2}{|l|}{ Edad (años) } \\
\hline Media & 30.4 \\
\hline Desviación estándar & 5.7 \\
\hline Rango & 23-39 \\
\hline \multicolumn{2}{|l|}{ Centro médico } \\
\hline Profamilia & $11(61.1)$ \\
\hline Clínica Medellín & $6(33.3)$ \\
\hline Clínica Prado & $1(5.6)$ \\
\hline \multicolumn{2}{|l|}{ Sexo } \\
\hline Mujer & $18(100 \%)$ \\
\hline Hombre & $0(0 \%)$ \\
\hline \multicolumn{2}{|l|}{ Raza } \\
\hline Caucásica & $0(0 \%)$ \\
\hline Mestiza & $18(100 \%)$ \\
\hline Negra & $0(0 \%)$ \\
\hline \multicolumn{2}{|l|}{ Ocupación } \\
\hline No empleada & $11(61.1)$ \\
\hline Empleada & $6(33.3)$ \\
\hline Independiente & $1(5.6)$ \\
\hline \multicolumn{2}{|l|}{ Residencia } \\
\hline Medellín & $10(55.6)$ \\
\hline Área metropolitana & $2(11.1)$ \\
\hline Resto Antioquía & $6(33.3)$ \\
\hline \multicolumn{2}{|l|}{ Aseguramiento } \\
\hline Contributivo & $12(66.7)$ \\
\hline Subsidiado & $6(33.3)$ \\
\hline \multicolumn{2}{|c|}{ Historia familiar de cáncer de mama } \\
\hline Sí & $4(22.2)$ \\
\hline No & $14(77.8)$ \\
\hline \multicolumn{2}{|l|}{ Consumo de tabaco } \\
\hline Sí & $1(5.6)$ \\
\hline No & $17(94.4)$ \\
\hline \multicolumn{2}{|c|}{ Fecha del último parto (años) } \\
\hline Media & 6.5 \\
\hline Desviación estándar & 5.3 \\
\hline Rango & 3-19 \\
\hline
\end{tabular}

fecha del último parto promedio fue de 6,5 años antes (DE: 5.3; rango: 3-19).

Las características clínicas se muestran en la tabla 2. El motivo de consulta más común fue cambios inflamatorios (12, 66.7\%), seguido de masa y mastalgia asociada (12, 66.7\%). El tiempo promedio de los síntomas fue de 20.4 meses (DE: 44.5; rango: 1-180). La afectación mamaria no mostró una lateralidad dominante y la localización más frecuente fue central o retroareolar $(10,55.6 \%)$, Los signos clínicos más frecuentes fueron engrosamiento cutáneo $(18,100 \%)$ y fístula o cicatriz (16, $88.9 \%)$. Solo 5 pacientes $(27.8 \%)$ 
Tabla 2. Características clínicas

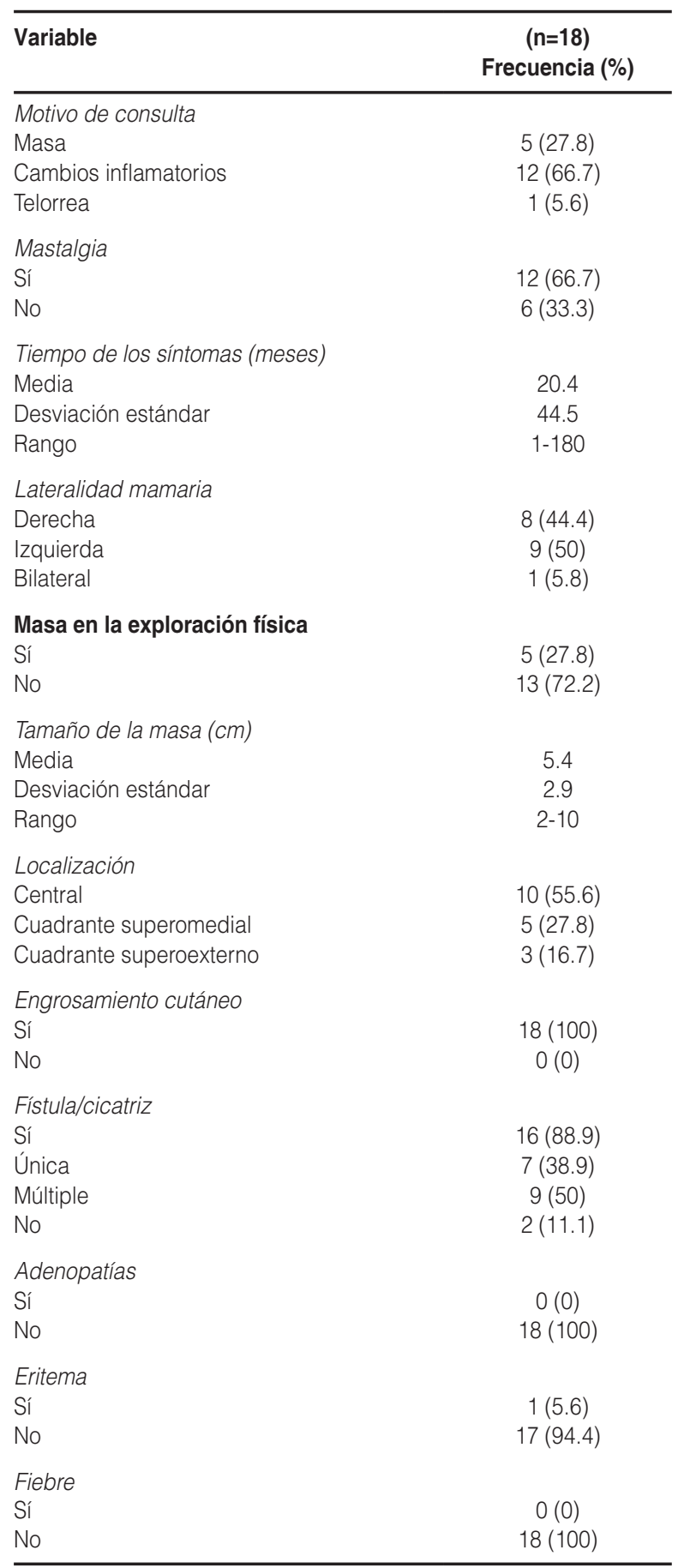

tenían masa y 1 (5.6\%) eritema en la exploración física. No hubo casos con adenopatías o fiebre.

\section{Estudios paraclínicos}

Los estudios paraclínicos se detallan en la tabla 3. Solo en una paciente $(5.6 \%)$ se solicitó la
Tabla 3. Estudios paraclínicos

\begin{tabular}{lc}
\hline Laboratorios & \\
\hline Variable & (n=18) \\
& Frecuencia (\%) \\
\hline Medición de & \\
prolactina & $0(0)$ \\
Sí. normal & $1(5.6)$ \\
Sí. anormal & $17(94.4)$ \\
no & \\
\hline Imagenología & \\
\hline Hallazgos ecografía & \\
Nódulo & \\
Quiste & $1(5.6)$ \\
Colección & $1(5.6)$ \\
Distorsión & $13(72.2)$ \\
Inespecífico & $1(5.6)$ \\
benigno & $2(11.1)$ \\
BI-RADS ecográfico & \\
0 & \\
1 & \\
2 & \\
3 & $0(0)$ \\
4 & $0(0)$ \\
5 & $8(44.4)$ \\
6 & $5(27.8)$ \\
Mamografía & $5(27.8)$ \\
No & 0 \\
\hline & 0 \\
& \\
& \\
& \\
& \\
& \\
&
\end{tabular}

determinación de prolactina y fue anormal. A todas los pacientes $(18,100 \%)$ se les realizó ecografía de mama y la categoría 2 del BI-RADS (Breast Imaging Reporting and Data System) fue la más frecuente. Únicamente en 2 casos (11.2\%) se realizó mamografía.

\section{Seguimiento}

Los datos del seguimiento se muestran en la tabla 4. El seguimiento promedio fue de 4.6 meses (DE: 4.7; rango: 1-17). En casi todos los casos se resolvió la enfermedad $(17,94.4 \%)$ y el tiempo de resolución promedio fue de 7 meses (DE: 8.2; rango: 2-36). Hubo 5 casos $(27.8 \%)$ con recurrencia, con un promedio de 2.7 episodios (DE: 2.2; rango: 1-6).

\section{Tratamiento}

Las modalidades de tratamiento se muestran en la tabla 5. La antibioticoterapia fue el tratamiento más utilizado $(13,72.2 \%)$ y la media de duración fue de 10 días (DE: 3.3; rango: 7-15), con múltiples antibióticos 
Tabla 4. Seguimiento

\begin{tabular}{lc}
\hline Variable & $\begin{array}{c}(\mathbf{n}=\mathbf{1 8}) \\
\text { Frecuencia (\%) }\end{array}$ \\
\hline Recurrencia & $5(27.8)$ \\
Sí & $13(72.2)$ \\
No & \\
N. ${ }^{\text {recurrencias }}$ & \\
Media & 2.7 \\
Desviación estándar & 2.2 \\
Rango & $1-6$ \\
Seguimiento (meses) & \\
Media & 4.6 \\
Desviación estándar & 4.7 \\
Rango & $1-17$ \\
Resolución & \\
Sí & $17(94.4)$ \\
No & $1(5.6)$ \\
Tiempo hasta resolución (meses) & \\
Media & \\
Desviación estándar & 7.0 \\
Rango & 8.2 \\
\hline
\end{tabular}

en su mayoría (8, 44.4\%); los antibióticos más usados fueron dicloxacilina, clindamicina y ciprofloxacino. El drenaje ecoguiado se realizó en 6 casos (33.3\%) y la mastotomía en 3 (16.7\%).

Los corticosteroides se usaron en 8 casos (44.4\%), con una dosis promedio de prednisona de $18 \mathrm{mg}$ (DE: 10.3; rango: 5-30) y una duración media de 5.4 semanas (DE: 3.7; rango: 3-12). El esquema utilizado en la mayoría fue de dosis alta $(0.5 \mathrm{mg} / \mathrm{kg}$ al día) con reducción de dosis gradual.

En 2 casos (11.1\%) se usó metotrexato y el tiempo promedio de tratamiento fue de 46.5 días (DE: 61.5; rango: 3-90).

La cirugía se realizó solo en 3 casos (16.7\%), de los cuales hubo 1 (5.6\%) mastectomía.

En las diferentes instituciones participantes, los algoritmos de abordaje diagnóstico y de tratamiento son discrecionales del médico tratante. Existe una tendencia a que el diagnóstico de mastitis granulomatosa se haga como enfermedad de exclusión, con confirmación histopatológica por inmunohistoquímica, y el tratamiento en la mayoría de los casos incluye antibioticoterapia antiestafilocócica en monoterapia o politerapia, y en pocos casos las pacientes son llevadas a cirugía. En la tabla 6 se muestra el análisis bivariado para la asociación entre resolución o recurrencia de la mastitis granulomatosa y distintos tipos de tratamiento.
Tabla 5. Tratamiento

\begin{tabular}{|c|c|}
\hline Variable & $\begin{array}{l}\quad(n=18) \\
\text { Frecuencia (\%) }\end{array}$ \\
\hline \multicolumn{2}{|l|}{ Antibióticos } \\
\hline Sí & $13(72.2)$ \\
\hline No & $5(27.8)$ \\
\hline \multicolumn{2}{|c|}{ Tiempo antibioticoterapia (dias) } \\
\hline Media & 10 \\
\hline Desviación estándar & 3.3 \\
\hline Rango & $7-15$ \\
\hline \multicolumn{2}{|l|}{ Antibióticos } \\
\hline Penicilina & $2(11.1)$ \\
\hline Macrólido & $1(5.6)$ \\
\hline Clindamicina & $2(11.1)$ \\
\hline Múltiples & $8(44.4)$ \\
\hline No se reporta & $5(27.8)$ \\
\hline \multicolumn{2}{|l|}{ Drenaje por ecografía } \\
\hline Sí & $6(33.3)$ \\
\hline No & $12(66.7)$ \\
\hline \multicolumn{2}{|l|}{ Mastotomía } \\
\hline Sí & $3(16.7)$ \\
\hline No & $15(83.3)$ \\
\hline \multicolumn{2}{|l|}{$N .^{\circ}$ drenajes } \\
\hline 1 & $18(100)$ \\
\hline 2 & $0(0)$ \\
\hline \multicolumn{2}{|l|}{ Tratamiento esteroide } \\
\hline Sí & $8(44.4)$ \\
\hline No & $10(55.6)$ \\
\hline \multicolumn{2}{|c|}{ Dosis diaria de prednisona (mg) } \\
\hline Media & 18 \\
\hline Desviación estándar & 10.3 \\
\hline Rango & $5-30$ \\
\hline \multicolumn{2}{|c|}{ Tiempo tratamiento esteroides (semanas) } \\
\hline Media & 5.4 \\
\hline Desviación estándar & 3.7 \\
\hline Rango & $3-12$ \\
\hline \multicolumn{2}{|l|}{ Metotrexato } \\
\hline Sí & $2(11.1)$ \\
\hline No & $16(88.9)$ \\
\hline \multicolumn{2}{|c|}{ Tiempo de tratamiento metotrexato (días) } \\
\hline Media & 46.5 \\
\hline Desviación estándar & 61.5 \\
\hline Rango & $3-90$ \\
\hline \multicolumn{2}{|l|}{ Cirugía } \\
\hline Sí & $3(16.7)$ \\
\hline Cuadrantectomía & $2(11.1)$ \\
\hline Mastectomía & $1(5.6)$ \\
\hline No & $15(83.3)$ \\
\hline
\end{tabular}

\section{Discusión}

La prevalencia de mastitis granulomatosa en el presente estudio fue del $2.12 \%$, similar a lo reportado por 
Tabla 6. Análisis bivariado para la asociación entre la resolución y la recurrencia de la mastitis granulomatosa y distintos tipos de tratamiento

\begin{tabular}{|c|c|c|c|c|c|c|c|}
\hline \multicolumn{4}{|c|}{ Resolución } & \multicolumn{4}{|c|}{ Recurrencia } \\
\hline Variable & OR & IC95\% & $p$ & Variable & OR & IC95\% & $p$ \\
\hline Antibioticoterapia & 1.25 & $0.80-1.93$ & 0.09 & Antibioticoterapia & 0.02 & $0.00-0.41$ & 0.00 \\
\hline $\begin{array}{l}\text { Drenaje } \\
\text { ecoguiado }\end{array}$ & 1.09 & $0.92-1.29$ & 0.46 & $\begin{array}{l}\text { Drenaje } \\
\text { ecoguiado }\end{array}$ & 1.71 & $1.06-2.76$ & 0.06 \\
\hline Mastotomía & 1.07 & 0.93-1.22 & 0.64 & Mastotomía & 8 & $0.53-120$ & 0.09 \\
\hline Esteroides & 1.11 & $0.90-1.36$ & 0.30 & Esteroides & 9 & $0.74-108$ & 0.06 \\
\hline Metotrexato & 1.06 & $0.94-1.21$ & 0.71 & Metotrexato & 3 & $0.15-59.8$ & 0.45 \\
\hline Cirugía & 1.07 & $0.93-1.22$ & 0.64 & Cirugía & 1.37 & $0.09-19.6$ & 0.81 \\
\hline
\end{tabular}

IC95\%: intervalo de confianza del 95\%; OR: odds ratio.

Baslaim, et al. ${ }^{5}$, quienes hallaron un $1.8 \%$. En los Estados Unidos de Norteamérica se reportó una incidencia del $0.37 \% \%^{6}$, y se han descrito mayores tasas en mujeres latinas y asiáticas ${ }^{5}$.

La edad media en esta serie fue de 30 años, con rango entre 23 y 39 años, similar a lo reportado por otros autores, como DeHertogh, et al. ${ }^{9}$ y Han, et al. ${ }^{7}$, en cuyas series la edad media se encontraba entre 20 y 49 años. La mayoría de las pacientes tienen antecedentes recientes de embarazo y lactancia ${ }^{4,5,10}$; en el presente estudio, la fecha promedio del último parto fue 6.5 años antes.

La presentación clínica más común en esta serie fueron los cambios inflamatorios de la mama, como inflamación, engrosamiento de la piel, dolor y formación de abscesos y fistulas. Puede afectar cualquier cuadrante, con mayor tendencia en la región subareolar, similar a lo reportado por Akcan, et al. ${ }^{14}$, pero en su estudio la masa fue el hallazgo más común, por lo que es necesario descartar el carcinoma de mama ${ }^{24,25}$. Otros diagnósticos diferenciales incluyen otras causas de inflamación granulomatosa por infecciones como tuberculosis, parasitarias o fúngicas, y no infecciosas como sarcoidosis, granulomatosis de Wegener, arteritis de células gigantes, poliarteritis o reacción a cuerpo extraño $0^{2,18,26,27}$.

En el presente estudio se realizó ecografía en los 18 casos y los hallazgos ecográficos más comunes fueron colección heterogénea, áreas de ecogenicidad con fístula cutánea y engrosamiento de la piel suprayacente. Las categorías BI-RADS 2-3 fueron las más frecuentes, de modo similar a lo reportado por otros autores ${ }^{28}$.

El tratamiento sigue siendo controvertido y no es universalmente aceptado; incluye el uso de antibióticos, corticosteroides, metotrexato y cirugía. En general, es una enfermedad autolimitada con un curso natural de 6 a 12 meses sin importar qué tratamiento se reciba, y en más de la mitad de las pacientes que no reciben ningún tratamiento se resuelve por completo ${ }^{16,29}$. En el presente estudio, el $94 \%$ se resolvieron totalmente en un periodo promedio de 7 meses; un porcentaje importante, el $28 \%$, presentaron recurrencia.

En este estudio se prefirió el manejo no quirúrgico (81.3\%). Algunos autores han sugerido un manejo conservador como el método de tratamiento más apropiado $^{30-33}$. El $72 \%$ de las pacientes recibieron antibióticos orales, por un diagnóstico inicial de mastitis infecciosa. Hovanessian Larsen, et al. ${ }^{3}$ reportan que la antibioticoterapia no fue útil y solo $6(3 \%)$ de sus pacientes tuvieron mejoría. El análisis bivariado del presente estudio no mostró beneficio; se puede argumentar que los antibióticos no deben tener un efecto significativo debido a que la patogenia no incluye la infección bacteriana ${ }^{3,34}$.

De acuerdo con la teoría autoinmunitaria, los esteroides son el tratamiento de primera línea. En esta serie, el $44 \%$ de las pacientes recibieron prednisona. Se reservó para la presentación clínica con síntomas avanzados o más graves, debido a que las dosis altas de esteroides pueden llevar a efectos secundarios importantes. El tiempo de administración en este estudio fue de 5 semanas, mientras que otros estudios reportan una duración promedio de 5 meses $^{9,14,35}$. Azlina, et al. ${ }^{17}$ hallaron tasas de recurrencia de hasta el $50 \%$. El análisis bivariado del presente estudio no demostró beneficio en la resolución ni en la reducción de la recurrencia. 
El metotrexato se utilizó en aquellas pacientes que fueron resistentes a la terapia con esteroides después de 3 meses, así como para la recurrencia después del inicio de la terapia con esteroides con respuestas variables $^{35,36}$. Akbulut, et al. ${ }^{35}$ analizaron retrospectivamente 541 casos de mastitis granulomatosa tratados con esteroides, metotrexato o ambos entre 1972 y 2010, y encontraron una alta tasa de recurrencia y también la aparición de efectos secundarios adversos. En el presente estudio, el análisis bivariado no demostró mejoría en la resolución ni en la disminución de la recurrencia.

Por otro lado, en cuanto a la intervención quirúrgica, cuando se usa comúnmente en la práctica clínica, se han informado tasas de recurrencia del $16 \%$ al $50 \%$, por lo que algunos autores recomiendan que la extirpación se haga con márgenes negativos ${ }^{15-17,37}$. En este estudio se realizó cirugía al $16.7 \%$ de las pacientes, y en un caso fue mastectomía. En la revisión de la literatura, las tasas de recurrencia variaron del $5.5 \%$ al $50 \%{ }^{31}$. En este estudio hubo recurrencia en 5 pacientes (27.8\%); en el análisis bivariado, la antibioticoterapia mostró una reducción significativa de la recurrencia.

\section{Limitaciones}

Las limitaciones de este estudio incluyen su naturaleza retrospectiva y que no se contó con el uso de anticonceptivos orales como variable.

\section{Conclusiones}

La mastitis granulomatosa es una enfermedad inflamatoria benigna de la mama, de causa desconocida, infrecuente y de difícil diagnóstico, que afecta a mujeres en edad fértil y generalmente se presenta con cambios inflamatorios, con mastalgia y en muchos casos con masa mamaria. El ultrasonido es útil para determinar las características de las masas y las colecciones abscedadas, para la toma de biopsias y para el drenaje percutáneo. El diagnóstico final requiere la confirmación histológica. El tratamiento puede ir desde la observación hasta el uso de corticosteroides, dejando la resección quirúrgica para pocos casos. La recurrencia reportada es alta y el seguimiento a largo plazo resulta esencial.

\section{Agradecimientos}

El autor agradece a Profamilia, Clínica Medellín y Clínica del Prado.

\section{Conflicto de intereses}

El autor declara no tener ningún conflicto de intereses.

\section{Responsabilidades éticas}

Protección de personas y animales. Los autores declaran que los procedimientos seguidos se conformaron a las normas éticas del comité de experimentación humana responsable y de acuerdo con la Asociación Médica Mundial y la Declaración de Helsinki.

Confidencialidad de los datos. Los autores declaran que han seguido los protocolos de su centro de trabajo sobre la publicación de datos de pacientes.

Derecho a la privacidad y consentimiento informado. Los autores han obtenido el consentimiento informado de los pacientes y/o sujetos referidos en el artículo. Este documento obra en poder del autor de correspondencia.

\section{Bibliografía}

1. Kessler E, Wolloch Y. Granulomatous mastitis: a lesion clinically simulating carcinoma. Am J Clin Pathol. 1972;58:642-6.

2. Lin $\mathrm{CH}$, Hsu CW, Tsao TY, Chou J. Idiopathic granulomatous mastitis associated with risperidone-induced hyperprolactinemia. Diagn Pathol. 2012;7:2.

3. Hovanessian Larsen LJ, Peyvandi B, Klipfel N, Grant E, lyengar G. Granulomatous lobular mastitis: imaging, diagnosis, and treatment. AJR Am J Roentgenol. 2009;193:574e81.

4. Brown KL, Tang PH. Postlactational tumoral granulomatous mastitis: a localized immune phenomenon. Am J Surg. 1979;138:326e9.

5. Baslaim MM, Khayat HA, Al-Amoudi SA. Idiopathic granulomatous mastitis: a heterogeneous disease with variable clinical presentation. World J Surg. 2007;31:1677-81.

6. Centers for Disease Control and Prevention. Idiopathic granulomatous mastitis in Hispanic women - Indiana, 2006-2008. MMWR Morb Mortal Wkly Rep. 2009;58:1317-21.

7. Han BK, Choe YH, Park JM, Moon WK, Ko YH, Yang JH, et al. Granulomatous mastitis: mammographic and sonographic appearances. AJR Am J Roentgenol. 1999;173:317- 20.

8. Breast Imaging Reporting and Data System (BI-RADS). (Consultado en julio de 2012.) Disponible en: www.birads.at/info.html.

9. DeHertogh DA, Rossof AH, Harris AA, Economou SG. Prednisone management of granulomatous mastitis. N Engl J Med. 1980;308:799-800.

10. Carmalt HL, Ramsey-Stewart G. Granulomatous mastitis. Med J Aust. 1981;1:356-9

11. Koray $O$, Ahmet D, Ozgur T. Granulomatous mastitis: clinical, pathological features, and management. Breast J. 2010;16:176.

12. Yau FM, Macadam SA, Kuusk U. The surgical management of granulomatous mastitis. Ann Plast Surg. 2010;64:9.

13. Afridi SP, Memon A, Shafig R. Granulomatous mastitis: a case series. J Coll Physicians Surg Pak. 2010;20:365.

14. Akcan A, Akyildiz H, Deneme MA, Akgun H, Aritas Y. Granulomatous lobular mastitis: a complex diagnostic and therapeutic problem. World J Surg. 2006;30:1403-9.

15. Tavassoli FA. Pathology of the breast. 2nd ed. NewYork: McGraw-Hill; 1999.

16. Lai EC, Chan WC, Ma TK, Tang AP, Poon CS, Leong HT. The role of conservative treatment in idiopathic granulomatous mastitis. Breast $\mathrm{J}$. 2005;11:454-6.

17. Azlina AF, Ariza Z, Arni T, Hisham AN. Chronic granulomatous mastitis: diagnosis and therapeutic considerations. World J Surg. 2003;27:515-8.

18. Memis A, Bilgen I, Ustun EE, Ozdemir N, Erhan Y, Kapkac M. Granulomatous mastitis: imaging findings with histopathologic correlation. Clin Radiol. 2002;57:1001e6. 
19. Imoto S, Kitaya T, Kodama T, Hasebe T, Mukai K. Idiopathic granulomatous mastitis: case report and review of the literature. Jpn J Clin Oncol. 1997;27:274a7.

20. Martínez-Parra D, Nevado-Santos M, Meléndez-Guerrero B, García-Solano J, Hierro-Guilmain CC, Pérez-Guillermo M. Utility of fine-needle aspiration in the diagnosis of granulomatous lesions of the breast. Diagn Cytopathol. 1997;17:108e14

21. Fletcher A, Magrath IM, Riddell RH, Talbot IC. Granulomatous mastitis: a report of seven cases. J Clin Pathol. 1982;35:941-5.

22. Galea MH, Robertson JF, Ellis IO, Elston CW, Blamey RW. Granulomatous lobular mastitis. Aust N Z J Surg. 1989:59:547-50.

23. Jorgensen MB, Neilsen DM. Diagnosis and treatment of granulomatous mastitis. Am J Med. 1992;93:97-101.

24. De Sanctis DP, Maglietta R, Amalfitano G, Loffredo D, Betta PG. Idiopathic granulomatous mastitis. Report of a case clinically and mammographically simulating breast carcinoma. Pathologica. 1994;86:222-3.

25. Tokunaga E, Kimura Y, Kitamura K, Maehara Y. Granulomatous lobular mastitis misdiagnosed as breast carcinoma. Breast J. 2004;10:261-2.

26. Bani-Hani KE, Yaghan RJ, Matalka II, Shatnawi NJ. Idiopathic granulomatous mastitis: time to avoid unnecessary mastectomies. Breast $\mathrm{J}$. 2004;10:318-22.

27. Seo HR, Na KY, Yim HE, Kim TH, Kang DK, Oh KK, et al. Differentia diagnosis in idiopathic granulomatous mastitis and tuberculous mastitis. J Breast Cancer. 2012;15:111e8.

28. Lacambra M, Thai TA, Lam CC, Yu AM, Pham HT, Tran PV, et al. Granulomatous mastitis: the histological differentials. J Clin Pathol. 2011;64:405-11.
29. Mahlab-Guri K, Asher I, Allweis T, Diment J, Sthoeger ZM, Mavor E. Granulomatous lobular mastitis. Isr Med Assoc J. 2015;17:476e480.

30. Freeman CM, Xia BT, Wilson GC, Lewis JD, Khan S, Lee SJ, et al. Idiopathic granulomatous mastitis: a diagnostic and therapeutic challenge. Am J Surg. 2017;214:701-6.

31. Kiyak G, Dumlu EG, Kilinc I, Tokaç M, Akbaba S, Gurer A, et al. Management of idiopathic granulomatous mastitis: dilemmas in diagnosis and treatment. BMC Surg. 2014;14:66.

32. Joseph KA, Luu X, Mor A. Granulomatous mastitis: a New York public hospital experience. Ann Surg Oncol. 2014;21:4159-63.

33. Oran E, Gürdal SÖ, Yankol Y, Öznur M, Calay Z, Tunacı M, et al. Management of idiopathic granulomatous mastitis diagnosed by core biopsy: a retrospective multicenter study. Breast J. 2013;19:411-8.

34. Aghajanzadeh M, Hassanzadeh R, Alizadeh Sefat S, Alavi A, Hemmati H, Esmaeili Delshad MS, et al. Granulomatous mastitis: presentations, diagnosis, treatment and outcome in 206 patients from the north of Iran. Breast. 2015;24:456-60.

35. Akbulut S, Arikanoglu Z, Senol A, Sogutcu N, Basbug M, Yeniaras E, et al. Is methotrexate an acceptable treatment in the management of idiopathic granulomatous mastitis? Arch Gynecol Obstet. 2011;284: 1189 e95.

36. Kim J, Tymms KE, Buckingham JM. Methotrexate in the management of granulomatous mastitis. A N Z J Surg. 2003;73:247e9.

37. Ocal K, Dag A, Turkmenoglu O, Kara T, Seyit H, Konca K. Granulomatous mastitis: clinical, pathological features, and management. Breast J. 2010;16:176e182. 